\title{
Social media and suicide prevention: A systematic review
}

Running title: Suicide prevention and social media

\section{Authors and affiliations:}

Jo Robinson $^{1}$, Georgina Cox $^{1}$, Eleanor Bailey ${ }^{1}$, Sarah Hetrick ${ }^{1}$, Maria Rodrigues ${ }^{2}$, Steve Fisher ${ }^{2}$, Helen $\operatorname{Herrman}^{1}$

1. Orygen Youth Health Research Centre, Centre for Youth Mental Health, University of Melbourne, 35 Poplar Road, Parkville, Victoria 3052, Australia.

2. Community Works Associates Pty Ltd, 313/757 Bourke Street, Docklands, Victoria 3008, Australia.

\section{Corresponding author:}

Jo Robinson, Orygen Youth Health Research Centre, Centre for Youth Mental Health, University of Melbourne, 35 Poplar Road, Parkville, Victoria 3052, Australia.

Tel: +61 $393422866 \quad$ Fax: +61 $393422941 \quad$ Email: jr@unimelb.edu.au

Word count: 4,964 (abstract $=265)$ 


\section{Abstract}

Aim: Social media platforms are commonly used for the expression of suicidal thoughts and feelings, particularly by young people. Despite this, little is known about the ways in which social media can be used for suicide prevention. The aim of this study was to conduct a systematic review to identify current evidence pertaining to the ways in which social media are currently used as a tool for suicide prevention.

Methods: Medline, psychInfo, Embase, CINHAL, and the Cochrane Library were searched for articles published between 1991 and April 2014. English language articles with a focus on suicide-related behaviour and social media were included. No exclusion was placed on study design.

Results: Thirty studies were included; four described the development of social media sites designed for suicide prevention, six examined the potential of social media in terms of its ability to reach or identify people at risk of suicide; 15 examined the ways in which people used social media for suicide prevention-related purposes; and five examined the experiences of people who had used social media sites for suicide prevention purposes. No intervention studies were identified.

Conclusion: Social media platforms can reach large numbers of otherwise hard-to-engage individuals, may allow others to intervene following an expression of suicidal ideation online, and provide an anonymous, accessible and non-judgmental forum for sharing experiences. Challenges include difficulties controlling user behaviour and accurately assessing risk, issues relating to privacy and confidentiality and the possibility of contagion. Social media appears to hold significant potential for suicide prevention; however additional research into its safety and efficacy is required.

Keywords: suicide, social media, review 


\section{Introduction}

The World Health Organisation (WHO) estimates that suicide is the 13th leading cause of death worldwide, and is the leading cause of death among those aged between 15 and 39. Globally, it is estimated to account for approximately one million deaths per year. The prevalence of non-fatal suicide-related behaviour, such as suicide attempt, is more common and may be up to 20 times higher than fatal suicidal behaviour, with an estimated 10 to 20 million non-fatal attempted suicides occurring every year worldwide ${ }^{[1]}$.

However despite its prevalence, limited evidence exists with regard to the efficacy of interventions designed to reduce suicide risk, including among young people ${ }^{[2]}$. This is the case in both clinical ${ }^{[3]}$ and school settings ${ }^{[4]}$.

The last decade has seen the internet become an increasingly powerful form of information and communication media, in particular among young people ${ }^{[5]}$. It is also becoming widely used in the treatment of a range of health-related problems, including adolescent depression and anxiety ${ }^{[6]}$. However to our knowledge, to date only one small pilot study has tested the effects of an internet-based program among suicidal young people, and found it to be safe, acceptable and potentially efficacious ${ }^{[7,8]}$.

The technological foundations of Web 2.0 extended the capacity of the internet by enabling users to not only receive information, but to create and exchange their own content, leading to the advent of social media websites. As such, social media has been defined as a group of mobile and internet-based applications that allow the creation and exchange of user-generated contents ${ }^{[9,10]}$. Systematic categorisation of social media is difficult due to the fact that cyberspace is constantly evolving, however commonly used forms of social media include social networking sites, such as Facebook; blogs and micro-blogs, such as Tumblr and Twitter; collaborative projects, such as Wikipedia; and content communities, such as YouTube ${ }^{[9]}$.

The growth of internet-based communication and social media has presented a new set of challenges for the field of suicide prevention. Indeed, concerns have long been expressed with regard to the safety of talking about suicide using more traditional forms of media. For example, articles that sensationalise suicide, contain explicit descriptions of means of suicide, or portray suicide as a legitimate solution to one's problems are believed to increase the risk of contagion, particularly among already vulnerable individuals ${ }^{[11,12]}$. Social media sites, which can be used for the expression of suicidal feelings and for the communication about suicide-related 
behaviour with others, may exacerbate this ${ }^{[13,14]}$. This has raised a series of questions regarding the ways in which we talk about suicide in online environments and how this may affect others ${ }^{[15]}$.

Despite the possible risks involved, social media’s increasing popularity, especially with young people who are often hard to engage in traditional forms of treatment ${ }^{[16]}$, and its potential as a tool for preventing and/or treating depression ${ }^{[17]}$, suggest it has potential to be used as a preventative tool ${ }^{[18]}$. For the most part, however, the extent of this potential remains unknown. Indeed, whilst systematic reviews exist that examine the effects of social media-based interventions on depression ${ }^{[17]}$, and the effects of internet-based interventions on suiciderelated behaviour ${ }^{[19]}$, none actually examine the effects of social media-based platforms on suicide-related outcomes.

Therefore the aim of this study was to conduct a systematic review in order to identify current evidence pertaining to the ways in which social media are currently being used as a tool for suicide prevention. As such, we specifically focussed on those studies that reported on the ways in which social media platforms are currently being used in preventative ways as opposed to studies that described the possible negative impacts social media may have.

\section{Methods}

Search strategy

An electronic literature search was conducted following the methodology set out in the Cochrane Collaboration Handbook for Systematic Reviews ${ }^{[20]}$. Medline, psychInfo, Embase, CINHAL, and the Cochrane Library were searched for articles published between 1991 till April 2014. The following keywords formed the basis of the search: (suicid* OR attempted suicid* OR Self-Injurious Behavior* OR DSH OR deliberate self-harm) AND (web* OR online* OR blog OR internet OR computer* OR chat* OR tech* OR dot* OR cyber* OR virtual OR surf OR electronic OR social media OR facebook OR twitter OR linkedin OR pinterest OR myspace OR google+ OR deviantart OR livejournal OR tagged OR Orkut). The top 10 most popular websites included in the search string were selected from the following site: http://www.forward.ph/blog/top-10-most-popular-socialnetworks-2013/. In addition, reference lists of all included studies, and previous literature reviews were hand searched. 
English language studies published in a peer-reviewed journal were included in the review. Studies had to focus on either suicide or suicide-related behaviour, and to focus on the development, or use, of a social media platform as a vehicle to support people at risk of suicide. Because this is an emerging field and we wanted to examine a broad range of studies no restriction was placed on study design.

Studies were excluded if: they did not have a preventative focus (for example studies that focused on the link between cyber bullying and suicide, or that reported on the association between social media use and suiciderelated behaviours); they reported on a non-interactive website; they examined the representation of suicide on the internet by analysing web pages retrieved through the ‘Google’ search engine; or if they focused on nonsuicidal self-injury only. Reviews and editorials were also excluded.

Included articles were then classified according to the research question the original study aimed to answer; these were:

1. What format do social media sites developed specifically for the purpose of suicide prevention take?

2. What is the potential of social media in terms of its ability to 'reach' and identify people at risk of suicide prevention?

3. How do people use social media sites for suicide prevention-related purposes? (This included sites that had been specifically developed for the purpose of suicide prevention, and already existing sites e.g. MySpace)

4. What are the experiences of people who use social media sites for suicide-related purposes?

\section{Data extraction}

Data were extracted by two study authors (GC and EB) with input from a third author (JR). Where possible, data pertaining to study design and aims, target population, study methodology, website format or content and presence or absence of moderation and safety protocols (where appropriate), participant or user characteristics, key findings or results, including limitations or challenges and study conclusions were extracted. A narrative synthesis of all data was conducted, as this was most appropriate given the nature of the included studies.

\section{Results}

Search results 
Search results are summarised in the Prisma Flow Chart in Figure 1 below. As can be seen, the initial search produced 652 results. One author (GC) removed duplicate papers and screened the titles and abstracts of all remaining papers for possible inclusion $(n=355)$. At this stage, any papers that did not meet the inclusion criteria for this review were excluded ( $n=269)$. The full text was retrieved for the remaining 86 papers. These were then screened for inclusion by two authors (GC and JR). Of these, 55 were excluded for the following reasons: 22 were review articles, conference proceedings, editorials or study protocols; 19 did not focus on the role of social media in suicide prevention; 6 focussed on the relationship between internet search terms and suicide-related behaviour; 4 described non-interactive sites; 2 focussed on non-suicidal self-injury; and 2 could not be obtained.

[Please insert Figure 1 about here]

The 1998 study by Baume ${ }^{[21]}$ was a publication based on an earlier paper published in 1997 by the same authors ${ }^{[22]}$ and contained additional discussion points. For the purpose of this review, the 1997 record was classed as a secondary publication to the 1998 paper.

Ultimately, 30 separate studies were included in the current review.

\section{Description of studies}

The studies included in the current review adopted a range of methodological approaches. These included: studies that examined the structure, format or operation of social media platforms; studies that mapped the linkages between or reach of various social media platforms; studies that analysed the content of social media sites or platforms using either qualitative and/or quantitative techniques; studies that administered either interviews or surveys to users of social media sites or platforms; individual case studies of people who had self harmed; and case series studies of people who had self harmed.

Studies were classified as per the research questions described above. The key characteristics of these studies are summarised in Tables 1-4.

1. What format do social media sites developed specifically for the purpose of suicide prevention take? 
Four studies sought to answer this question. Two of these specifically targeted suicidal individuals and comprised email, instant chat, and online forums as well as information-based articles and lists of support organisations ${ }^{[23,24]}$. One site targeted the staff and students of an American university ${ }^{[25]}$. This site comprised a static website containing information, emergency contact information, and an online course, and used social media platforms MySpace and Facebook in order to increase the visibility of, and provide information about, the program. The final study targeted mental health professionals that work with suicidal people ${ }^{[26]}$. This site took the form of an interactive website and chat room that enabled users to access professional development resources, to give and receive peer support, and to disseminate, exchange, and share information.

[Please insert Table 1 about here]

Together these studies found that the websites described were well used. Benefits to individuals at risk included the potential for emotional support, reduced feelings of isolation and the 24-hour nature of help ${ }^{[23,24]}$, whilst benefits of the site targeting professionals resulted in the exchange knowledge and expertise that would not otherwise have been shared ${ }^{[26]}$. Challenges included difficulties controlling member participation, difficulties accurately assessing the emotional state of participants online ${ }^{[23]}$, the emotional burden on volunteers and the potential for technological problems ${ }^{[24]}$.

What is the potential of social media in terms of its ability to 'reach' and identify people at risk of suicide prevention?

In terms of reach, one study specifically set out to answer this research question. This study conducted a series of Monte Carlo simulations in order to examine how well MySpace could be used to target prevention research towards lesbian, gay, and bisexual adolescents ${ }^{[27]}$. This study found that a peer-driven preventative intervention delivered via MySpace was able to reach more than 18,000 individuals, a significantly larger sample than could be reached via more traditional methods, thus highlighting the potential reach of suicide prevention activities delivered via social media.

However other studies also reported findings relevant to this question. For example, the study by Fu and colleagues primarily sought to examine responses to a wrist-cutting picture posted on a Chinese microblogging site ${ }^{[28]}$. However in addition to high numbers of positive messages, they also reported large numbers of 'reposts' indicating that networks such as these can be activated quickly in emergency situations, thereby 
highlighting the potential for social networking sites to be used to support and detect at-risk individuals and to possibly facilitate intervention in suicide attempts. This was echoed by Belfort et al's examination of people who post suicide notes online, which found that the majority of participants who disclosed their suicidality electronically subsequently presented to services for help because a peer had seen the information and either shared it with an adult or encouraged the young person to seek help ${ }^{[29]}$. This latter study also highlights the potential that social media can offer in terms of early identification of individuals at risk.

Two case studies elicited similar findings. The first of these reviewed a case report in which a clinician discovered the suicidal ideation of a client on Facebook and took actions that led to a hospital admission ${ }^{[30]}$. The second presented a case study involving a suicide note posted on Facebook by a person who subsequently completed suicide ${ }^{[31]}$. The authors discuss the ethical challenges raised by these scenarios, including issues relating to privacy and confidentiality, clinical judgment, and informed consent, plus the potential risk that 'copycat' events may occur. However, despite these challenges both studies highlight how the public nature of suicide-related social media posts can provide opportunities for intervention.

On a broader level two studies examined social networking sites in order to identify suicide-related posts and then examined how these related to overall suicide rates in the respective countries ${ }^{[32,33]}$. Together these studies found evidence to suggest that suicide-related posts on social networking sites were significantly associated with suicide rates. For example, Jashinsky and colleagues ${ }^{[33]}$ found a significant association between frequency of suicide posts on Twitter and data on actual suicide rates in the USA, whilst Won and colleagues ${ }^{[32]}$ found suicide-related posts on a Korean social networking site to be significantly associated with nationwide suicide rates. In both studies the authors concluded that it might be feasible to use these social media platforms to monitor suicide trends, collect suicide-related data, and to deliver suicide prevention activities.

[Please insert Table 2 about here]

2. How do people use social media sites for suicide prevention-related purposes?

This was the largest category with 15 studies. Eleven studies examined the ways in which people used specifically designed online suicide forums ${ }^{[14,21,28,34-40]}$, including those designed for people bereaved by 
suicide $^{[41]}$. Two studies primarily focussed on the ways in which moderators responded to messages posted in the forums ${ }^{[42,43]}$, and the final two studies examined both of these things ${ }^{[44,45]}$.

[Please insert Table 3 about here]

Overall these studies reported a range of benefits to the individual; these included the supportive atmosphere created by site moderators and other forum members, as well as the accessibility and utility of these sites ${ }^{[40-45]}$. Benefits also included the opportunity that online environments offer individuals to talk about their feelings and experiences with similar others without being judged ${ }^{[40,41,44]}$. Additionally, Fu and colleagues ${ }^{[28]}$ found that the majority of responses to a wrist-cutting picture posted online contained messages that were caring, showed empathy, or called for help. Only one study had an overall negative focus citing the potential dangers of contagion when communicating suicidal intent online ${ }^{[21]}$.

These studies found that suicidal and non-suicidal forum members could be distinguished on the basis of their writing style, with suicidal members more frequently distancing themselves from direct dialogue and social involvement and displaying a more depressogenic attribution style ${ }^{[34,35,39]}$; and one found that individuals used the sites primarily to validate their suicidal identity rather than to genuinely seek help ${ }^{[37]}$.

Finally, three studies examined the ways in which site moderators respond to forum users. Two of these examined the responses of volunteer moderators to suicidal messages posted on an online forum ${ }^{[42,43]}$. In the first, they aimed to reveal and explore strategies used by the forum moderators when responding to suicidal posts ${ }^{[42]}$ and in the second they compared these responses with those offered by lay individuals ${ }^{[43]}$. The authors found that whilst both moderators and lay individuals tended to offer emotional support, moderators used this technique more often. Moderators also used response strategies of empowerment, interpretation, and cognitive change that were rarely used by lay individuals. In contrast lay individuals more frequently included selfdisclosure in their responses.

The last of these studies described the responses of the site moderator and group members to the suicide of an online forum member ${ }^{[45]}$. The responses were described as being similar to those expected in a real-life 
community, and nobody expressed support of the suicide or reported engaging in suicidal behaviour in response. The author reported facing challenges regarding how to represent the death in a way that would facilitate mourning whilst not sensationalising it and hence risk causing contagion. The author recommended that online forums should have threaded asynchronous discussions or separate chat rooms, and searchable archives in order to allow people to 'work through' their grief in the event of the suicide of a forum member. Memorial threads or distinct memorial pages can be useful and should follow recommendations for print media in order to minimise contagion.

3. What are the experiences of people who use social media sites for suicide-related purposes?

Five studies investigated the experiences of individuals that use social media-based platforms for suicide prevention purposes either via interview or via a survey. Three of these studies focused on vulnerable individuals ${ }^{[46-48]}$ and two focussed on individuals who had been bereaved by suicide ${ }^{[49,50]}$. Three studies focused on interactive online activity in general and did not specify the particular online platforms being used

${ }^{[46,48,51]}$; one study focused on an unnamed discussion forum for suicidal people ${ }^{[47]}$, and the final study focussed on Parents of Suicide; an online support group for parents who have been bereaved by suicide ${ }^{[52]}$.

[Please insert Table 4 about here]

Overall these studies reported that people who post suicidal messages online do so for a number of reasons. Several studies identified that people use these forums in order to gain information and to connect and share their experiences with others with similar problems, in what is perceived to be a positive, readily accessible and supportive manner ${ }^{[46-48]}$. For example, a study by Harris, McLean, and Sheffield ${ }^{[48]}$ of individuals who go online for suicide-related purposes found that $70 \%$ of these individuals used online forums and they generally found them to be supportive and useful. They also reported that individuals who went online for suicide-related purposes were less likely to seek help offline than suicide-risk individuals who did not go online for suiciderelated purposes, and they were less likely to seek help offline than the comparison group.

One survey-based study found that very few users went online to find a suicide partner or information about suicide methods, and the majority reported a reduction in suicidal ideation after joining suicide forum ${ }^{[47]}$. Caution was expressed however that in some cases suicide was portrayed as a legitimate solution to one’s problems and some users displayed a distrust of conventional psychiatric services and institutions. 
Three studies examined the experience of using social media platforms upon individuals bereaved by suicide.

Overall these reported that participants had positive views of using online support groups, and gained considerable benefits with key factors being the ability to hear from others with similar experiences in an anonymous, non-judgmental environment ${ }^{[51,52]}$. The availability, including the 24-hour nature of the sites, was also reported as being beneficial ${ }^{[51,52]}$. Feigelman and colleagues ${ }^{[52]}$ also reported that individuals who used an internet support group had higher levels of depression, suicidal ideation, and grief, and were more likely to have experienced unhelpful responses from family and friends, than those who used a face-to-face support group.

\section{Discussion}

Key findings

This review identified 30 unique studies that had a primary focus on the ways in which social media platforms could be used for the purpose of suicide prevention. Four studies described the development of social media sites designed for suicide prevention, six examined the potential of social media in terms of its ability to reach or identify people at risk of suicide; 15 examined the ways in which people used social media for suicide prevention-related purposes; and five examined the experiences of people who had used social media sites for suicide prevention purposes. No studies reported on the development of, or findings from, an intervention study using social media for suicide prevention purposes.

Overall, study design and quality was variable. The majority of studies included in the review were descriptive and cross sectional in nature, and in most cases examined the utility of a single website or forum thus limiting the generalisability of the findings from the individual studies. However despite the limitations in terms of study design the findings from the studies retrieved by the current review are of interest. Overall the findings appeared to be largely positive with a number of key advantages of using social media for this purpose highlighted. These included the ability of social media platforms to reach large numbers of otherwise hard to engage people ${ }^{[27]}$, the visibility of expressions of suicidal ideation posted online, which can in turn allow others to intervene in, and potentially prevent, a suicide attempt ${ }^{[28-31]}$, and its acceptability in terms of providing an anonymous, accessible and non-judgmental forum for sharing experiences with others and for both providing and receiving support; this was the case for both people who were suicidal themselves ${ }^{[38,40,46,48]}$ and for those who had been bereaved 
by suicide ${ }^{[41,51,52]}$. Sites were generally governed by an ethical code of conduct and successfully moderated by trained volunteers who received professional supervision ${ }^{[24,36,44]}$.

Suicidal individuals reported using these platforms to seek peer support as opposed to professional help, and very few people used the sites to seek information about suicide methods or suicide partners ${ }^{[38,40]}$. Despite this, some concerns and challenges were raised. These included the possibility that utilising social media platforms as opposed to offline help and support could serve to further marginalise people from mainstream society ${ }^{[46]}$, the normalising of suicide-related behaviour as a response to one's problems ${ }^{[40]}$, difficulties controlling user behaviour and accurately assessing participants' emotional state online ${ }^{[23,24]}$, and the possibility of contagion [21].

\section{Limitations}

When considering the implications of these findings certain limitations must be borne in mind. Firstly, whilst the search strategy employed was as thorough as possible, some studies may have been overlooked, particularly those that were not available in English.

It is also important to note that because we were interested in getting a broad understanding of all studies pertaining to social media and its potential role in suicide prevention, no restrictions were placed on study design. Whilst this is not unusual in reviews where evidence (in particular from randomised controlled trials) is considered to be limited ${ }^{[4]}$, or in research examining the potential impact of social media ${ }^{[17]}$ it did mean that the nature and quality of included studies varied significantly and that developing a classification system for the studies was challenging. For example, several of the studies focussed on users from a single discussion forum, thus limiting the generalisability of the study findings. Similarly the cross sectional nature of some of the survey-based studies prevents us from making any inferences regarding causal relationships between social media use and changes in suicide-related outcomes. However, research into the impact of social media platforms is an emerging field, with inherent methodological challenges (returned to below), therefore it was considered important to include these studies in the review as they still provide us with some indication of the potential benefits of using social media in suicide prevention. 
Finally because we were interested in examining the role of social media in suicide prevention we only included those studies with a preventative focus. As a result a number of studies, for example those that examined the relationship between social media use and suicide risk or the role of cyber-bullying in suicide-related behaviour, were excluded and this may appear to give the results a more positive emphasis than would otherwise have been the case.

Implications

Despite the limitations cited above, this review highlights a number of potential benefits offered by social media platforms when it comes to suicide prevention. These primarily centre on the reach, accessibility, and nonjudgmental and anonymous nature of such platforms. However these same benefits also present numerous ethical and methodological challenges for clinicians and researchers respectively.

In clinical terms, whilst the anonymous nature of social media increases its appeal among users, it makes it difficult for clinicians to conduct adequate risk assessments or to trace people considered to be at immediate risk ${ }^{[23]}$. Ethical challenges also exist in terms of duty of care, privacy and confidentiality ${ }^{[30]}$. These are significant challenges for clinicians and may partially explain the apparent disconnect between the ways in which young people use social media (i.e. primarily for peer-to-peer support) and the ways in which professionals appear to use it, (i.e. for the delivery of information and awareness raising) ${ }^{[53]}$. The need for clear protocols and ethical standards for the delivery and evaluation of suicide prevention activities online has been acknowledged ${ }^{[54,55]}$. Most of the individual sites described here were governed by ethical codes of conduct, however work is currently underway in Australia ${ }^{[56]}$ and in the U.S. ${ }^{[57]}$ to develop detailed guidelines for the online delivery of suicide prevention activities and their implementation will hopefully be an important step towards enhancing the safe delivery of suicide prevention initiatives using the internet.

The findings from this review highlight the utility of social media both as an alternative to face-to-face treatment for some, but also as an adjunct to treatment for others. Therefore, if we are to fully harness the benefits afforded by social media, professionals need to work with consumers in order to better incorporate the positive aspects of social media into day-to-day clinical practice ${ }^{[36,46]}$. 
Just as above with regard to clinical practice, it is the very aspects of social media platforms that make them appealing that also present numerous methodological challenges for researchers. For example, the rapidly evolving nature, reach and anonymity of social media render the collection of reliable data almost impossible using current methodological approaches ${ }^{[19]}$. More flexible and iterative approaches that promote maximum stakeholder involvement and have the capacity to produce more timely and relevant research findings methodologies are therefore required. Such approaches exist in other fields ${ }^{[58]}$ and could readily be applied to suicide prevention.

Despite these challenges, social media platforms are clearly popular for seeking support for suicide-related feelings. This is not only exemplified by the studies retrieved here, but has been previously identified by another study in which an extensive internet search was conducted in order to detect ways in which social media platforms are being used for suicide prevention ${ }^{[59]}$. This phenomenon can also been seen in web pages such as the popular 'Coming Together to Prevent Youth Suicide’ Facebook page ${ }^{[60]}$, which was established by community members in response to a group of suicides by young people and now has over 20,000 members.

There is clearly a demand for social media to be used in the prevention of suicide. The question facing professionals is how can this be done safely? Biddle and colleagues ${ }^{[61]}$ discussed the potential for the development of legislation governing internet content to minimise the amount of harmful content available. However, this may be challenging to implement and the effects would be difficult to measure. Several authors have also advocated for service providers to utilise search optimisation strategies designed to boost their visibility, thus maximising the likelihood that helpful, rather than harmful sites, are the first to appear in an internet search ${ }^{[61-64]}$. One way of doing this may be to add certain 'meta-tags' or keywords that people may use when searching for suicide methods for example, to suicide prevention websites in order to increase the likelihood of retrieving a helpful site when method-related search terms are used ${ }^{[64]}$. Other initiatives, include the removal of pro-suicide sites and links, and partnerships with organisations such as the Samaritans in the UK and Ireland to launch reporting systems that allow users to report concerns they have about other users who may be expressing suicidal ideation ${ }^{[65]}$.

Whilst these are positive initiatives, there is, to date, a lack of published literature evaluating their effectiveness. That there are no studies of this nature and that no intervention studies were identified by our search is 
disappointing but not surprising. Researchers have repeatedly reported a lack of high quality studies that have tested the effects of interventions on suicide-related outcomes, in particular using randomised controlled designs $[3,4,66]$, including online ${ }^{[19,45,67]}$, and this was reflected in the studies included in the current review. Social media is becoming increasingly popular, particularly with young people, and there is growing evidence supporting the acceptability, efficacy and cost effectiveness of delivering depression prevention and treatment programs online ${ }^{[6]}$, although evidence is less conclusive in terms of the actual benefits of social media platforms ${ }^{[17]}$. Applying appropriate methodologies to testing the use of social media platforms for both depression and suicide prevention purposes is therefore warranted ${ }^{[17,45]}$ and is a logical next step. Examples of interventions that could be readily tested include the dissemination of specific suicide prevention messaging either delivered universally, or to those identified as being vulnerable by the sorts of messages that they post, and/or the distribution of mobile applications designed to reduce suicide risk such as safety planning or problem solving tools. However this may require a move away from the traditional RCT. The feasibility of the RCT to suicide prevention has been questioned previously ${ }^{[68]}$, and this would seem to be increasingly relevant given the move towards online application of interventions.

\section{Conclusion}

Whilst more high quality studies are clearly needed, in particular intervention studies, the findings from this review suggest that social media platforms appear to enable people at risk of suicide to access information, support and counselling, and to share their experiences in a flexible, timely and readily accessible format. They also allow these individuals to simultaneously receive and provide support in what is perceived to be a safe and non-judgemental environment, thus enabling them to create unique and positive identities in a way that they may not otherwise have been able to do.

Whilst the potential hazards should not be ignored, it appears that, if used carefully, social media could play an important role in suicide prevention, providing a useful adjunct to more traditional forms of treatment. 


\section{Statement of contributorship}

JR conceived the study, determined the inclusion and exclusion criteria, designed the classification system, supervised and assisted with screening and data extraction, interpreted the data and prepared the manuscript. GC developed the search strategy and ran the search. She also assisted with screening, development of the classification system, data extraction and preparation of the manuscript. EB assisted with development of the classification system, data extraction and preparation of the manuscript. SH assisted with development of the classification system, interpretation of the study findings and preparation of the manuscript. MR assisted with an earlier version of the search, screening and data extraction. She also provided comments on the manuscript. SF and $\mathrm{HH}$ provided assistance in the initial stages of designing the study, assisted with interpretation of the findings and provided comments on the manuscript. All authors have reviewed and are in agreement with the content of this manuscript.

\section{Acknowledgments}

The study was conducted at Orygen, the National Centre of Excellence in Youth Mental Health and the Centre for Youth Mental Health at the University of Melbourne, and Community Works (Australia). It was conducted under the auspice of the Young and Well Cooperative Research Centre Australia (CRC) and the World Health Organization (WHO) Collaborating Centre in Mental Health Melbourne, in consultation with the WHO Western Pacific Regional Office of the World Health Organization. The authors would like to acknowledge Dr Xiangdong Wang for his efforts initiating this study and Professor Jane Pirkis for her comments on the manuscript.

\section{Declaration of conflicting interests}

The authors declare that there is no conflict of interest

\section{Funding}

This work was supported by the Young and Well Cooperative Research Centre and Orygen Youth Health Research Centre. 


\section{References}

1. World Health Organisation. World Suicide Prevention Day Media Release: Suicide Prevention (Supre) 2009; Available from: http://www.who.int/mental health/prevention/suicide/suicideprevent/en.

2. Robinson J, Pirkis J. Research priorities in suicide prevention: an examination of Australianbased research 2007-11. Aust Health Rev 2013; 38(1): 18-24.

3. Robinson J, Hetrick S, Martin C. Preventing suicide in young people: Systematic review. Aust NZ J Psychiat 2011; 45(1): 3-26.

4. Robinson J, Cox G, Malone A, Williamson M, Baldwin G, Fletcher K, et al. A systematic review of school based interventions aimed at preventing, treating, and responding to, suicide-related behaviour in young people. Crisis 2013; 34(3): 162-82.

5. Ewing S, Thomas J, Schiessl J. CCl Digital Futures Report: The internet in Australia. Melbourne: ARC Centre for Excellence for Creative Industries and Innovation Institute for Social Research, Swinburne Univerity of Technology, 2008.

6. Calear AL, Christensen H. Review of internet-based prevention and treatment programs for anxiety and depression in children and adolescents. Med J Aust 2010; 192(11 Suppl): S12-4.

7. Robinson J, Hetrick S, Cox G, Bendall S, Yung AR, Pirkis J. The safety and acceptability of delivering an online intervention to secondary students at risk of suicide: findings from a pilot study. Early Interv Psychiatry 2014. doi: 10.1111/eip.12136

8. Robinson J, Hetrick SE, G. C, Bendall S, Yuen HP, Yung AR, et al. Can an Internet-based intervention reduce suicidal ideation, depression and hopelessness among secondary school students? Results from a pilot study. Early Interv Psychiatry 2014. doi: 10.1111/eip.12137 9. Kaplan AM, Haenlein M. Users of the world, unite! The challenges and opportunities of social media. Bus Horizons 2010; 53(1): 59-68.

10. Boyd DM, Ellison NB. Social network sites: definition, history, and scholarship. J ComputMediat Comm 2007; 13(1): 210-30.

11. Lee DTS, Chan KPM, Yip PSF. Burning charcoal: a novel and contagious method of suicide in Asia. Arch Gen Psychiatry 2002; 59(3): 293-4.

12. Sisask M, Varnik A. Media roles in suicide prevention: a systematic review. Int J Environ Res Public Health 2012; 9(1): 123-38.

13. Becker K, Mayer M, Nagenborg M, El-Faddagh M, Schmidt MH. Parasuicide online: can suicide websites trigger suicidal behaviour in predisposed adolescents? Nord J Psychiat 2004; 58(2): 111-4.

14. Cash S, Thelwall M, Peck S, Ferrell J, Bridge J. Adolescent suicide statements on MySpace. Cyberpsychol Behav Soc Netw 2013; 16(3): 166-74.

15. Pirkis J, Robinson J. Improving our understanding of youth suicide clusters. Lancet Psychiatry 2014; 1(1): 5-6.

16. Hunt I, Kapur N, Robinson J, Shaw J, Flynn S, Bailey H, et al. Suicide within 12 months of mental health service contact in different age and diagnostic groups: national clinical survey. Brit J Psychiatr 2006; 188(2): 135-42.

17. Rice S, Goodall J, Hetrick SE, Parker AG, Gilberton T, Amminger P, et al. Online and social networking interventions for the treatment of depression in young people: a systematic review. J Med Internet Res 2014; 16(9): e206.

18. Robinson J, McGorry P. Time to break the silence on a last taboo. The Age. 2009 Tue 25 Aug. 19. Lai MH, Maniam T, Chan LF, Ravindran AV. Caught in the web: a review of web-based suicide prevention. Journal of Medical Internet Research 2014; 16(1): e30.

20. Higgins JPT, Green S. Cochrane Handbook for Systematic Reviews of Interventions Version 5.0.2. Collaboration TC. 2009.

21. Baume $P$, Rolfe $A$, Clinton $M$. Suicide on the Internet: a focus for nursing intervention? AJMHN 1998; 7(4): 134-41. 
22. Baume $\mathrm{P}$, Cantor $\mathrm{CH}$, Rolfe $\mathrm{A}$. Cybersuicide: the role of interactive suicide notes on the Internet. Crisis 1997; 18(2): 73-9.

23. Gilat I, Shahar G. Suicide prevention by online support groups: an action theory-based model of emotional first aid. Arch Suicide Res 2009; 13(1): 52-63.

24. Barak A. Emotional support and suicide prevention through the internet: a field project report. Comput Hum Behav 2007; 23(2): 971-84.

25. Manning J, Vandeusen K. Suicide prevention in the dot com era: technological aspects of a university suicide prevention program. J Am College Health 2011; 59(5): 431-3.

26. Penn DL, Simpson L, Edie G, Leggett S, Wood L, Hawgood J, et al. Development of ACROSSnet: an online support system for rural and remote community suicide prevention workers in Queensland, Australia. Health Informatics J 2005; 11(4): 275-93.

27. Silenzio VMB, Duberstein PR, Tang W, Lu N, Tu X, Homan CM. Connecting the invisible dots: reaching lesbian, gay, and bisexual adolescents and young adults at risk for suicide through online social networks. Soc Sci Med 2009; 69: 469-74.

28. Fu KW, Cheng Q, Wong PWC, Yip PSF. Responses to a self-presented suicide attempt in social media. Crisis 2013; 34(6): 406-12.

29. Belfort EL, Mezzacappa E, Ginnis K. Similarities and differences among adolescents who communicate suicidality to others via electronic versus other means: a pilot study. Adolescent Psychiat 2012; 2(3): 258-62.

30. Lehavot K, Ben-Zeev D, Neville RE. Ethical considerations and social media: a case of suicidal postings on Facebook. J Dual Diagn 2012; 8(4): 341-6.

31. Ruder TD, Hatch GM, Ampanozi G, Thali MJ, Fischer N. Suicide announcement on Facebook. Crisis 2011; 32(5): 280-2.

32. Won HH, Myung W, Song GY, Lee WH, Kim JW, Carroll BJ, et al. Predicting national suicide numbers with social media data. PloS One 2013; 8(4): e61809.

33. Jashinsky J, Burton SH, Hanson CL, West J, Giraud-Carrier C, Barnes MD, et al. Tracking suicide risk factors through Twitter in the US. Crisis 2014; 35(1): 51-9.

34. Barak A, Miron O. Writing characteristics of suicidal people on the internet: a psychological investigation of emerging social environments. Suicide Life-Threat 2005; 35(5): 507-24.

35. Fekete $\mathrm{S}$. The internet - a new source of data on suicide, depression and anxiety: a preliminary study Arch Suicide Res 2002; 6(4): 351-61.

36. Gilat I, Shahar G. Emotional first aid for a suicide crisis: comparison between telephonic hotline and internet. Psychiatr 2007; 70(1): 12-8.

37. Horne J, Wiggins S. Doing being 'on the edge': managing the dilemma of being authentically suicidal in an online forum. Sociol Health III 2009; 31(2): 170-84.

38. Ikunaga A, Nath SR, Skinner KA. Internet suicide in Japan: a qualitative content analysis of a suicide bulletin board. Transcult Psychiatry 2013; 50(2): 280-302.

39. Kupferberg I, Gilat I. The discursive self-construction of suicidal help seekers in computermediated discourse. Commun Med 2012; 9(1): 23-35.

40. Westerlund M. Talking suicide: online conversations about a taboo subject. NORDICOM Review 2013; 2(35): 35-46.

41. Schotanus-Dijkstra M, Havinga P, van Ballegooijen W, Delfosse L, Mokkenstorm J, Boon B. What do the bereaved by suicide communicate in online support groups?: A content analysis. Crisis 2013; 35(1): 27-35.

42. Gilat I, Tobin Y, Shahar G. Offering support to suicidal individuals in an online support group. Arch Suicide Res 2011; 15(3): 195-206.

43. Gilat I, Tobin Y, Shahar G. Responses to suicidal messages in an online support group: comparison between trained volunteers and lay individuals. Soc Psychiatry Psychiatr Epidemiol 2012; 47(12): 1929-35.

44. Greidanus E, Everall RD. Helper therapy in an online suicide prevention community. Brit J Guid Couns 2010; 38(2): 191-204. 
45. Hsiung RC. A suicide in an online mental health support group: reactions of the group members, administrative responses, and recommendations. Cyberpsychol Behav 2007; 10(4): 495500.

46. Baker D, Fortune S. Understanding self-harm and suicide websites: a qualitative interview study of young adult website users. Crisis 2008; 29(3): 118-22.

47. Eichenberg C. Internet message boards for suicidal people: a typology of users. Cyberpsychol Behav 2008; 11(1): 107-13.

48. Harris KM, McLean JP, Sheffield J. Examining suicide-risk individuals who go online for suicide-related purposes. Arch Suicide Res 2009; 13(3): 264-76.

49. Feigelman W, Gorman BS, Chastain Beal K, Jordan JR. Internet support groups for suicide survivors: A new mode for gaining bereavement assistance. Omega: Journal of Death and Dying 2008; 57(3): 217-43.

50. Chapple A, Ziebland S. How the Internet is changing the experience of bereavement by suicide: a qualitative study in the UK. Health (London, England : 1997) 2011; 15(2): 173-87. $10.1177 / 1363459309360792$

51. Chapple A, Ziebland S. How the internet is changing the experience of bereavement by suicide: a qualitative study in the UK. Health 2011; 15(2): 173-87.

52. Feigelman W, Gorman B, Chastain B, Jordan J. Internet support groups for suicide survivors: a new mode for gaining bereavement assistance. Omega-J Death Dying 2008; 57(3): 217-43.

53. Robinson J, Rodrigues M, Fisher S, Herrman H. Suicide and Social Media Study: Report of the Stakeholder Consultation. Melbourne, Australia: Orygen Youth Health Research Centre, Community Works, 2014.

54. Birbal R, Maharajh HD, Birbal R, Clapperton M, Jarvis J, Ragoonath A, et al. Cybersuicide and the adolescent population: challenges of the future? IJAMH 2009; 21(2): 151-9.

55. Kerkhof AJFM, Mishara BL. Promising practices, future prospects and research agenda. In: Mishara BL, Kerkhof AJFM, editors. Suicide prevention and new technologies: Evidence based practice. New York, U.S.A.: Palgrave MacMillan; 2013.

56. Young and Well Cooperative Research Centre HloMH. Outcomes report: National roundtable on social Media, suicide prevention and young people in Australia. Melbourne, Australia: Young and Well Cooperative Research Centre and the Hunter Institute of Mental Health, 2013.

57. Reidenberg D. Best practice tools for online safety. International Association for Suicide Prevention 2013 World Congress; 25/09/2013; Oslo, Norway2013.

58. Riley TW, Glasgow RE, Etheredge L, Abernethy AP. Rapid, responsive, relevant (R3) research: a call for a rapid learning health research enterprise. Clin Transl Med 2013; 2(1): 10.

59. Robinson J, Rodrigues M, Fisher S, Herrman H. Suicide and Social Media Study: Report of the Internet Search. Melbourne, Australia: Orygen Youth Health Research Centre, Community Works, 2014.

60. Coming Together to Prevent Youth Suicide. [Facebook page] [26/08/2014]; Available from: https://www.facebook.com/groups/preventingyouthsuicide/?fref=ts.

61. Biddle L, Donovan J, Hawton K, Kapur N, Gunnell D. Suicide and the internet. Brit Med J 2008; 336(7648): 800-2.

62. Krysinska K, Andriessen K. Online support and resources for people bereaved through suicide: what is available? Suicide Life-Threat 2010; 40(6): 640-50.

63. Kemp CG, Collings SC. Hyperlinked suicide: assessing the prominence and accessibility of suicide websites. Crisis 2011; 32(3): 143-51.

64. Till B, Niederkrotenthaler T. Surfing for suicide methods and help: Content analysis of websites retrieved with search engines in Austria and the United States. Journal of Clinical Psychiatry 2014; 75(8): 886-92.

65. Luxton DD, June JD, Fairall JM. Social media and suicide: a public health perspective. Am J Public Health 2012; 102 (Suppl 2): S195-200. 
66. Robinson J, Pirkis J, Krysinska K, Niner S, Jorm AF, Dudley M, et al. Research priorities in suicide prevention in Australia: a comparison of current research efforts and stakeholder-identified priorities. Crisis 2008; 29(4): 180-90.

67. Jacob N, Scourfield J, Evans R. Suicide prevention via the internet: a descriptive review. Crisis 2014; 35(4): 261-7.

68. Goldney R. Suicide prevention: A pragmatic review of recent studies. Crisis 2005; 26: 128-40. 


\section{University Library}

\section{- M M I N E R VA A gateway to Melbourne's research publications}

Minerva Access is the Institutional Repository of The University of Melbourne

\section{Author/s:}

Robinson, J;Cox, G;Bailey, E;Hetrick, S;Rodrigues, M;Fisher, S;Herrman, H

Title:

Social media and suicide prevention: a systematic review

Date:

2016-04-01

Citation:

Robinson, J., Cox, G., Bailey, E., Hetrick, S., Rodrigues, M., Fisher, S. \& Herrman, H. (2016). Social media and suicide prevention: a systematic review. EARLY INTERVENTION IN PSYCHIATRY, 10 (2), pp.103-121. https://doi.org/10.1111/eip.12229.

Persistent Link:

http://hdl.handle.net/11343/59201 